\title{
Characterization of the nonlinear elastic behavior of chinchilla tympanic membrane using micro-fringe projection
}

\author{
Junfeng Liang ${ }^{a}$, Huiyang Luo ${ }^{a}$, Zachary Yokell ${ }^{b}$, Don U. Nakmali ${ }^{b}$, Rong Zhu Gan ${ }^{b}$, and \\ Hongbing $\mathrm{Lu}^{\mathrm{a}}{ }^{*}$ \\ aDepartment of Mechanical Engineering, The University of Texas at Dallas, Richardson, TX \\ 75080, USA \\ bSchool of Aerospace and Mechanical Engineering, The University of Oklahoma, Norman, OK \\ 73019, USA
}

\begin{abstract}
The mechanical properties of an intact, full tympanic membrane (TM) inside the bulla of a fresh chinchilla were measured under quasi-static pressure from $-1.0 \mathrm{kPa}$ to $1.0 \mathrm{kPa}$ applied on the TM lateral side. Images of the fringes projected onto the TM were acquired by a digital camera connected to a surgical microscope and analyzed using a phase-shift method to reconstruct the surface topography. The relationship between the applied pressure and the resulting volume displacement was determined and analyzed using a finite element model implementing a hyperelastic $2^{\text {nd }}$-order Ogden model. Through an inverse method, the best-fit model parameters for the TM were determined to allow the simulation results to agree with the experimental data. The nonlinear stress-strain relationship for the TM of a chinchilla was determined up to an equibiaxial tensile strain of $31 \%$ experienced by the TM in the experiments. The average Young's modulus of the chinchilla TM from ten bullas was determined as approximately $19 \mathrm{MPa}$.
\end{abstract}

\section{Keywords}

chinchilla; tympanic membrane; micro-fringe projection; static pressure; hyperelastic model

\section{INTRODUCTION}

An eardrum or tympanic membrane (TM) couples acoustic waves from ambient air in the ear canal to the middle ear; it is a key component for transmitting sound pressure to ossicular chains. The function of the TM can be affected by ambient pressure, which changes widely from a few pascal $(\mathrm{Pa})$ to a few $\mathrm{kPa}$. In some extreme cases, for instance, under blast in conflict zones, the overpressure can be as high as $100 \mathrm{kPa}$, which could cause damage to the TM (Ritenour, et al., 2008). As TM deforms under different ambient pressures, the

\footnotetext{
*Corresponding author. Tel: (972)883-4647; (972)883-4655 (Fax). hongbing.lu@ utdallas.edu.

Publisher's Disclaimer: This is a PDF file of an unedited manuscript that has been accepted for publication. As a service to our customers we are providing this early version of the manuscript. The manuscript will undergo copyediting, typesetting, and review of the resulting proof before it is published in its final citable form. Please note that during the production process errors may be discovered which could affect the content, and all legal disclaimers that apply to the journal pertain.
} 
transmission of sound energy across the middle ear can be significantly altered (Dirckx, et al., 1991; Volandri, et al., 2011; Ghadarghadar, et al., 2013; Thornton, et al., 2013; Greff, et al., 2014a, 2014b; Rosowski, et al., 2014). In efforts made to understand the effect of ambient pressure on TM function for the sound transmission, the mechanical response of TMs has been investigated under various static pressures. The deformation of a TM, induced by either negative or positive pressure in the middle ear, was measured using shadow moiré technique on human temporal bones (Dirckx, et al., 1991). The TM vibration under different middle-ear pressures was measured on gerbil ears (Lee, et al., 2001; Rosowski, et al., 2002), where the alterations of acoustic stiffness and impedance by static pressures were observed. The stiffening of a TM under the repetitive pressure loading from habitual sniffing was investigated on gerbil using shadow moiré (von Unge, et al., 2009).

In addition to experimental investigations, finite element methods (FEM) have been used to study the sound transmission in the middle ear under various static pressures. The effect of geometrical nonlinearity was reported on the movement of a cat eardrum under static pressures on TM (Ladak, et al., 2006). The middle ear transfer function was also analyzed under various static pressures on a human middle ear (Wang, et al., 2007). It is noted that, the fidelity of the simulation results depends, to a large extent, on the accuracy of the mechanical properties of a TM, as a function of pressure.

The mechanical properties of TMs have been measured using numerous experimental techniques. The viscoelastic properties of a human TM were measured under tension using dynamic mechanical analysis (Cheng, et al., 2007) and acoustic pressure using laser Doppler vibrometery (LDV) (Gan, et al., 2010; Zhang, et al., 2010). A miniature split Hopkinson tension bar was used to measure the dynamic properties of human TMs under high strain rates (Luo, et al., 2009a, 2009b, 2016). Another method used was nanoindentation, it has been used to measure the mechanical properties of different quadrants of a TM. The linear viscoelastic properties of TMs were reported using nanoindentation (Huang, et al., 2008). The method was also used to measure both in-plane and out-of-plane mechanical properties at different locations of a TM (Daphalapurkar, et al., 2009). In all these methods, strip or cut TM specimens were used. This approach causes the collagen fibers in the radial or circumferential directions in pars tensa to shrink (O'Connor, et al., 2008), and alters the physiological condition of TM, leading potentially to erroneous results. To circumvent this problem, it is necessary to employ a new method to measure the mechanical properties for the full, intact TM, which is the focus of this paper.

Full-field measurement methods have been used in the last several years to measure the mechanical responses of a TM. Using LDV and stroboscopic holography, deformations of a human TM were measured with acoustic loading, and viscoelastic properties were determined through a hybrid method allowing FEM simulated umbo displacement to agree with the experimental measurement (De Greef, et al., 2014a, 2014b). Another full-field method to probe mechanical properties was developed to measure the TM elastic properties (Aernouts, et al., 2010; Buytaert, et al., 2009) using geometric moiré and indentation loading. The geometric moiré was used to determine the surface topography while the indentation was applied; the mechanical response of the TM was simulated by FEM to determine the elastic properties and the viscoelastic properties under low frequencies 
(Aernouts, et al., 2012a, 2012b, 2012c) using an inverse problem solving scheme. The Young's modulus was measured as approximately $20 \mathrm{MPa}$ by nano/micro-indentation (Aernouts, et al, 2010, 2012a, 2012b; Hesabgar, et al., 2010; Soons, et al., 2010; Salamati, et al., 2012). Both methods measure TM properties under indentation loading, which is applied on a small local region; that is a loading situation that is different from the condition under normal hearing or under blast, in which air pressure is applied on the entire TM. In addition, for the latter method, the contact nature and the localization of force applied on a small region could impose challenges to maintain the indenter positioning under increasing load. There is also a potential issue on convergence in the analysis considering a contact mechanics problem. An alternative computer-based method was developed; in which case, the pressure was used instead of indentation (Ghadarghadar, et al., 2013). In that method, the Young's modulus of TM was estimated by minimizing the difference in displacements over the entire TM calculated from model with that measured from experiment. The replacement of indentation loading with pressure loading simplified the experimental setup.

Ghadarghadar, et al. (2013) showed a rather good agreement between the computational displacement and experimental results on the pars tensa of the TM, but not on the pars flaccida (a small portion of the dataset). They also pointed out that the material property of pars flaccida can be significant different from pars tensa. It is noted that, the nonlinearity of the mechanical behavior of TM under different pressures has not been considered.

In this paper, we provide a non-contact, full-field optical method on the measurement of mechanical properties of an intact TM under quasi-static pressure. An inverse method combining experimental and numerical investigation is used to determine the mechanical properties of a chinchilla TM. The TM inside a bulla is pressurized while its topography is determined by a full-field micro-fringe projection technique. Volumetric displacement is then calculated from the topography. FEM with a nonlinear material model is applied to model the topography of TM under pressure, to provide the simulated relationship between pressure and volume displacement that is consistent with experiment. The nonlinear mechanical properties of TM under different quasi-static pressures are thus determined.

\section{METHOD}

\subsection{Micro-fringe projection technique}

A micro-fringe projection technique was used to determine the deformed surface topography of the TM under a prescribed static pressure. In micro-fringe projection, a grating is projected onto an object and the image of the projected fringe on the surface of the object is acquired by a digital camera. Another image of fringe projected onto a reference plane under the same setup is also acquired. The object image is subsequently digitally superimposed with the reference image to generate interferometry (Ortiz, et al., 2003, 2005). Virtual shifting is conducted by utilizing five phase-shifted images of the original image to calculate the phase difference between reference plane and the object from the interferometry map (Ortiz, et al., 2005).

The inverse tangent function outputs phase angle within the interval $[-\pi, \pi]$ with $2 \pi$ discontinuity at the end of the period. In order to determine the surface profile from the direct output, phase angle has to be unwrapped. A quality bins algorithm is used to unwrap 
the phase map for surface profile reconstruction (Ghiglia, et al., 1998). In the case where the projection is telecentric, the out-of-plane position $Z$ is determined from the phase angle difference $\Delta \Phi$ for any point on the object surface:

$$
Z=\frac{p h}{d} \frac{\Delta \Phi}{2 \pi}
$$

where $h$ is the distance between the camera and the object, and $d$ is the distance between the camera and the light source, as shown schematically in Figure 1(a). In the actual situation, it is difficult to measure accurately these parameters directly from the apparatus. A calibration procedure is thus used to determine the ratio between $h$ and $d$ in Eqn (1) (Ortiz, et al., 2003). A cone with known dimensions that approximately match the features (radius and depth) of the TM was used for calibration. By comparing the phase map with the known geometry, the ratio of $h$ to $d$ was determined.

\subsection{Experimental setup}

Figure 1(b) shows the actual experimental setup. The chinchilla bulla was placed on a gimbal holder attached to a temporal bone bowl, which allowed the orientation of the TM surface to be adjusted for micro-fringe projection as well as for observation by a camera. A set of X-, Y- and Z- ( $X Y Z)$ linear translation stages was used to hold the temporal bone bowl, to position the TM within the field of the projected fringes and field of view of the camera. A micro-fringe projector, including a set of lenses, grating, and fiber optic light source was used to project fringes onto the TM in the bulla. The projector consisted of a 100 W fiber optic lamp, two condenser lenses (Edmund Optical Sci., \#89-038), a grating and an objective lens (Fujinon Photo Optical Co., 611374). The focal length of the objective lens was adjustable so that an in-focus pattern of equidistant pitch fringes was projected onto the reference plane and the object. The grating used had a square wave transmission profile, namely the Ronchi rulings (Edmund Optical Sci., \#58-777) with pitch density of 20 cycle/mm. A digital camera (Nikon D7000, $4928 \times 3264$ pixels) was attached to a beam splitter on a surgical microscope (Carl Zeiss, OPMI-1) with an objective lens of $250 \mathrm{~mm}$. The microscope head was connected to a finely adjustable arm mounted on a movable stand, which allowed optical axis of the microscope to remain perpendicular to the sample holder plane. The projection lens of micro-fringe projector was located about $88 \mathrm{~mm}$ away from the microscope objective lens; an angle of approximately $19^{\circ}$ was formed between the axis of the microscope-camera assembly and axis of the projector. The combination of a finer grating and a smaller angle generally produces higher sensitivity than a coarser grating and a larger angle. In addition, the distance between the objective lens in Figure 1 and the object should be at least one order of magnitude larger than the height of the object (von Unge, et al., 1993). The height of chinchilla TM was about $2 \mathrm{~mm}$, which was much smaller than the working distance $(250 \mathrm{~mm})$ of the microscope. Therefore, the telecentric condition was satisfied.

A pressure monitoring system was used to load the specimen with either positive or negative static pressure. The system consists of two three-way stopcocks, a $20 \mathrm{~mL}$ syringe and a water manometer with a resolution of $2.5 \mathrm{~mm}$ water bar (Dwyer Instruments, \#1235). A 
three-way stopcock serves as a valve to control the pressure applied on the specimen; it allows for releasing and applying pressure to the specimen, and locking up the pressure in the specimen. Another three-way stopcock, serving as pressure direction control, was utilized to switch between positive pressure and negative pressure applied on the specimen.

\subsection{Sample preparation}

Ten TMs of adult chinchillas weighing between 535 grams to 855 grams, without middle ear diseases were used. Chinchilla was used because the diameter and the shape of its TM are close to human. The study protocol was approved by the Institutional Animal Care and Use Committee (IACUC) of the University of Oklahoma and met the guidelines of the National Institutes of Health. After examination with an otoscope, the chinchilla was sacrificed with ketamine $(100 \mathrm{mg} / \mathrm{kg} \mathrm{IM})$ and xylazine $(10 \mathrm{mg} / \mathrm{kg} \mathrm{IM})$ injected directly to the heart. An intact temporal bone or bulla was harvested from the skulls 10 minutes post mortem. The bulla wall was opened widely from the middle ear side and both the cochlea and the stapes along with the tensor tympani muscle were removed until the medial side of TM was fully exposed (Figure 2a). In order to maintain the geometry of the TM and simplify the boundary conditions for modeling, the malleus-incus complex was immobilized by applying a droplet of gel type superglue (Superglue, Co., Fast ${ }^{\mathrm{TM}}$ ) between the incus section and the petrous wall behind. A polyvinyl chloride (PVC) tubing with 3/16 inches inner diameter, 1/4 inches outer diameter, and about 3 inches long, was inserted into the ear canal and hermetically fixed on the bulla by applying two-part epoxy (Illinois Tool Works Inc, Devcon and 5 Minute) at the entrance of the ear canal. The two-part epoxy was mixed and cured for 2 minutes before it was applied on the bulla to ensure that it was viscous and would not flow into the middle ear. The outer end of the tubing was then connected to the pressure monitoring system for applying static pressure on the TM from the lateral side (Figure 2b). The overall time for sample preparation for each bulla in an experiment in this section was about 45 minutes. In order to protect the TM from desiccation, during the process of opening the bulla, a droplet of saline solution was applied on the TM every 5 minutes. Also, during the curing period of the two-part epoxy, a small piece of Kim wipe paper saturated with saline solution was used to cover the TM.

Similar to shadow moiré, fringe projection requires the object to have a diffusive reflecting surface. Therefore the medial side of TM was coated with a thin layer of titanium oxide in saline solution $(100 \mathrm{mg} / \mathrm{ml})$ to provide good reflection. Titanium oxide was chosen because it is a typical material used in cosmetics and a thin layer of the coating is not anticipated to affect the mechanical response of the TM (Dirckx, et al., 1997).

\subsection{Measurement procedures}

After the bulla specimen was prepared, it was mounted on the gimbal sample holder. The bulla was first secured with molding clay to adjust coarsely the position of the bulla so that TM faced the microscope and then fixed with the use of screwed arms of the holder; and the orientation of the bulla was finely adjusted to allow a full view of the TM without any shadow. The aforementioned PVC tubing was connected to the first three-way stopcock in the pressure monitoring system, and the stopcock was switched to change-pressure mode. Pressure was then applied to the bulla manually through the syringe in the pressure 
monitoring system. As a TM, like other soft tissue, is a viscoelastic material (Fung, 1993; Ladak, et al., 2004), preconditioning must be carried out to allow the specimen to give consistent results. Each bulla was preconditioned by applying a small pressure with magnitudes less than $100 \mathrm{~Pa}$ by five cycles prior to test. A positive pressure was induced by pumping compressed air into bulla from ear canal; as such, the pressure on the medial side of the TM was lower than that applied on the lateral side of the TM. A negative pressure was induced by a vacuum in the ear canal; in such a case the pressure on the medial side of the TM was higher than that applied on the lateral side. In each cycle in preconditioning, a negative pressure was applied first to the bulla, and then the pressure was increased to a positive value, and finally the pressure was released back to zero. Both positive and negative pressures were applied to the bulla for measurement of the response of the TM to pressure to determine the mechanical response of the TM. The magnitude of pressure applied was between 0 to $1.0 \mathrm{kPa}$ with an increment of $0.125 \mathrm{kPa}$ inside the bulla with stopcock set to lock-up mode. A constant pressure was maintained at each step. The entire measurement took about 2 minutes for each sample; therefore no special care was needed to maintain moisture in the TM.

At each state of pressure, including the zero-pressure state, an image was acquired by a digital camera attached to the microscope. For each image, reconstruction of TM surface was conducted using the method described in Section 2.1. The reconstructed TM surface profiles were then used to calculate the volume displacement based on the surface topography under pressure and the surface profile under the zero-pressure state.

\subsection{Finite element simulations}

The FEM software, ANSYS-15 was used for simulations of chinchilla TM under quasi-static pressure. A FEM model was established using the surface typography of the TM determined at the zero-pressure state. For each specimen, an individualized FEM model was established and the volume displacement under static pressure was calculated. Simulations were conducted by selecting the material parameters in the Ogden hyperelastic constitutive law until the volume displacement obtained from the FEM model matched well with the corresponding experimental data. The surface topography under the zero-pressure state, reconstructed from the micro-fringe projection, was converted to a three-dimensional model using the computer-aided design (CAD) software, SolidWorks 2013. Since the TM thickness is small compared with its major or minor axis, TM was modeled as a shell with a thickness of $10 \mu \mathrm{m}$ (Ghadarghadar, et al., 2013; Wang, et al., 2002). The CAD model was then converted to a shell model for simulations in ANSYS. The boundary and the location of malleus were determined by the optical image of the TM sample. Malleus was constructed using SolidWorks as a part of the TM assigned with properties of a bone (with $10 \mathrm{GPa}$ Young's modulus, and 0.2 Poisson's ratio). The outer boundary (annulus tympanicus) of the TM was fixed for all degrees of freedom (no translations or rotations), and a uniform pressure in the range of $0 \mathrm{kPa}$ to $1.0 \mathrm{kPa}$ was applied from the medial side (negative pressure) or lateral side (positive pressure), corresponding to the pressure used in experiments. 
Each meshed FEM model has nearly 10,000 4-node tetrahedral shell elements (shell-181) as shown in Figures 3(a) and 3(b); the boundary determined from optical image as shown in Figure 3(c) was used in FEM simulation. In practice, due to the continuation between epithelial layer of the TM and annulus, there is about 1 to 2 pixels of uncertainty for determining the boundary in the annulus area; this uncertainty induced less than $2 \%$ variation for the total area of TM. The uncertainty can be higher at the pars flaccida area. However, since the area of pars flaccida is less than 1/15 of pars tensa (Vrettakos, et al., 1988) and the displacement in pars flaccida area is usually much smaller than that of pars tensa (Ghadarghadar, et al., 2013), the uncertainty on the identification of the entire boundary is expected to induce less than $3 \%$ of error in the determination of volume displacement. Experimental data do not show any abrupt change of deformation in the neighborhood of pars flaccida area, therefore, only pars tensa was considered in both reconstruction and the FEM modeling. The medial and posterior views of a meshed TM model are shown in Figures 3(a) and 3(b), respectively, without showing the boundary conditions and the applied pressure. Figure 3(c) shows the medial-lateral views of the TM surface topography with boundary conditions. The outer boundary (annulus tympanicus) of the TM was fixed for all degrees of freedom (no translations or rotations) and a uniform pressure in the range of -1.0 to $1.0 \mathrm{kPa}$, was applied from the medial side (negative pressure) or lateral side (positive pressure), same as the pressure values used in experiments.

To simulate the pressure - volume deformation response observed in experiments, FEM analysis with the use of an appropriate constitutive model was conducted. Since a TM is essentially a biomaterial in rubbery state, a hyperelastic model traditionally developed for materials in the rubbery state such as elastomers was used in this study, to describe the constitutive behavior of a chinchilla TM under tension. The pressure-volume displacement response of TM shows behavior similar to an elastomer (Aerts, et al., 2010), including stiffening after reaching a certain pressure, the Ogden model was used to describe the mechanical behavior of TM under large deformations. The Ogden strain energy potential is given as:

$$
U=\sum_{i=1}^{N} \frac{2 \mu_{i}}{\alpha_{i}}\left(\lambda_{1}^{\alpha_{i}}+\lambda_{2}^{\alpha_{i}}+\lambda_{3}^{\alpha_{i}}\right), i=1,2, \ldots N
$$

where $N$ is the number of terms; $\lambda_{j}(j=1,2,3)$ are the principle stretch ratios; $\mu_{i}$ and $\alpha_{i}$ are constants. In this study, $N$ was taken as 2 . In order to determine the model parameters $\mu_{i}$ and $\mathrm{a}_{i}$, an inverse problem-solving scheme was used by allowing the FEM simulated TM volume displacement data to match the measured values under a given pressure. The procedures are described as follows.

1. Give vector of initial values, $p$ containing $\mu_{i}$ and $a_{i}$, as $p^{T}=\left[\mu_{1}, a_{1}, \mu_{2}, a_{2}\right]$.

2. Generate a FEM model for the TM, consistent with the geometry of the TM under the zero-pressure state. The TM is assigned with hyperelastic parameters (Ogden model). The out-of-plane displacement (i.e., displacement perpendicular to the image plane of the camera), and height $Z$ of each node was obtained. 
3. Interpolate nodal displacement obtained in Step 2 to the background grid with point density identical to the pixel density of images obtained in experiments using the bilinear interpolation scheme.

4. Calculate the volume displacement from the out-of-plane displacement of the background grid, and compare it with the experimental volume displacement data, which is calculated as:

$$
\Delta V=\iint_{\Omega}\left[z(x, y)-z_{0}(x, y)\right] \mathrm{d} x \mathrm{~d} y
$$

where, $z(x, y)$ and $z_{0}(x, y)$ are the height profiles under a finite pressure and the zero pressure, respectively, $\Omega$ is the area enclosed by the annulus. Optimization of a cost function is used to determine the Ogden parameters using $\Delta V$ at different pressures. The cost function is defined as:

$$
f=\sum_{i=1}^{M}\left(\Delta V_{i}^{e x p}-\Delta V_{i}^{F E M}\right)^{2}
$$

where $\Delta V_{i}^{\text {exp }}$ is the volume displacement obtained in experiment under a pressurized state, $\Delta V_{i}^{F E M}$ is the corresponding FEM simulated volume displacement, $M$ is the number of pressurized states.

5. Update $p$ using a gradient descent algorithm, and repeat Steps (1) through (4) until $f<0.05 \mathrm{~mm}^{6}$. The equation for gradient descent is given as:

$$
p^{\prime}=p-\gamma \nabla f(p)
$$

where $\gamma$ is the learning rate, set to be $0.1, \nabla f(p)$ is a vector of discrete form of gradient of $f$ with 4 elements, where each element is the difference of the cost function $f$ when one parameter in $p$ changes by 0.1 .

In this study, the initial parameters are set to the parameters for human (Cheng, et al., 2007). A range of initial values in the neighborhood of this set of initial parameters has been tried and it was found that within this range, the results converge to the same solution.

\section{RESULTS}

\subsection{Reconstruction of the TM surface under pressure}

The TM surface topography under different pressures was obtained from the micro-fringe projection system. Figure 4 shows the pressure-volume displacement relationships for five cycles of preconditioning at small pressure levels with amplitudes less than $0.1 \mathrm{kPa}$. Since chinchilla TMs are thin and fragile, some of the TMs ruptured during the process of loading. Therefore the pressure range for those samples was smaller than other samples. The 
preconditioning has significantly reduced the hysteresis: the difference in volume displacement between loading and unloading is in general less than $10 \%$ after preconditioning (Figure 4(b)). After five cycles of preconditioning, the pressure-volume displacement curves were gradually converged and the hysteresis loops became stable. It indicates that the mechanical behavior became steady, thus the TM was ready for characterization of the nonlinear elastic behavior. Figure 5(a) shows the TM image under projected micro-fringes before reconstruction of the surface. The four sections of TM, namely superior, posterior, inferior, anterior, and umbo are shown and marked as S, P, I, A and U, respectively. Figure 5(b) shows a typical surface height color contours of TM under the zero-pressure state from the reconstruction. Figures 5(c) and 5d show the corresponding $z$-displacement, $U_{3}$ contours under pressures. For the purpose of illustration, in the interest of space, only two cases are shown here: TM under $-1.0 \mathrm{kPa}$ and $1.0 \mathrm{kPa}$ pressures.

Under $-1.0 \mathrm{kPa}$, the edge of a TM close to inferior-posterior region has nearly zero displacement. Like other rodent, the manubrium of chinchilla is a thin bony edge, with thickness decreasing from about $1.0 \mathrm{~mm}$ at superior of annulus to roughly $0.1 \mathrm{~mm}$ at umbo. However, the region of low displacement is relatively large, which covers more than $1 / 3$ of the TM area around the manubrium. Likewise, around the annulus ring, a ring of low displacement region with width about $1.0 \mathrm{~mm}$ can be also observed. The maximum displacement is found to locate at the ring-shaped belt concentric with the annulus and about $1.5 \mathrm{~mm}$ from annulus ring covering the posterior, inferior and anterior. This probably stems from the thickness distribution of the TM, which is thicker at location around the bony boundary and thinner at location away from the bony boundary (Gea, et al., 2010). Similar displacement distribution is also seen in Figure 5(d) under positive pressure $1.0 \mathrm{kPa}$.

However, the region with low displacement under a positive pressure is small around the manubrium. The displacement at location close to the annulus increases smoothly to maxima at the location about $2.5 \mathrm{~mm}$ away from the annulus and then gradually decreases to zero. Due to the complex cone-shape geometry, a TM under positive and negative pressures exhibits different responses.

\subsection{Mechanical response of TM to various static pressures}

Using Eqn (3), the pressure-volume displacement relationship can be calculated from the $Z$ displacement, $U_{3}$ profile. Figure 6(a) shows the pressure as a function of the volume displacement, plotted in terms of the curves from the experimental results of ten chinchilla TMs with intact immobilized malleus-incus complex attached. It is noted that, results from some TMs have smaller range of volume displacement values due to the rupture of these TMs in experiment. To distinguish the TM samples, markers were used at certain data points. The pressure-volume displacement curves exhibit strong nonlinearity. The increasing stress with the increase of strain indicates the stiffening behavior of the chinchilla TM, and therefore the alternation of stiffness as pressure increases. This is likely due to the stiffening of the collagen fibers in the soft tissue. At the initial stage of the loading, collagen fibers are relaxed, showing a linear behavior. As the loading increases, the collagen fibers tend to align in the loading direction in response to the increasing load, to provide change in stiffness. 
Due to the nearly conical geometry of TM, volume displacements show significant difference between negative and positive pressures as shown in Figure 6. The deformation under positive pressure is larger than the deformation under negative pressure. The asymmetry of volume-displacement over positive/negative pressures is consistent with that was observed from TMs of other mammals, such as gerbil (Gea, et al., 2010), cat (Funnell et al., 1995), and human (Gaihede et al., 2007). Figure 6(b) shows a curve-fitting of the average pressure-volume displacement curve between FEM results and experimental data for ten TMs. Two extreme cases are also plotted in Figure 6(a), which are the best cases of fitting (smallest average error at each pressure point) for one of the animals and the worst case of fitting (largest average error at each pressure point) for another animal. The error is less than $10 \%$ between FEM and experimental data, indicating that the Ogden model is appropriate to describe the TM mechanical behavior. The model parameters are obtained under both positive and negative pressures. The dimensions of the TM and the material property parameters for Ogden model of each TM are listed in Table 1.

\subsection{Young's modulus of TM}

Since the TM is under biaxial stress state when a pressure is applied on it. An equibiaxial stress-strain relationship is used to describe the behavior of TM. The equibiaxial form of $N^{\text {th }}$-order Ogden model is given as (Ogden, 1972)

$$
T_{B}=\sum_{i=1}^{N} \frac{2 \mu_{i}}{\alpha_{i}}\left(\lambda_{B}^{\alpha_{i}-1}-\lambda_{B}^{-2 \alpha_{i}-1}\right), i=1,2, \ldots N
$$

where $T_{B}$ is the equibiaxial stress; $\lambda_{B}$ is the equibiaxial stretch ratio, and $\lambda_{B}=1+\varepsilon_{B}$, with $\varepsilon_{B}$ being the equibiaxial strain. Under an equibiaxial stretch, assuming incompressibility of the TM, the principal stretch ratios $\lambda_{i}(i=1,2,3)$ are given as $\lambda_{1}=\lambda_{2}=\lambda_{B}, \lambda_{3}=\lambda_{B}^{-2}$. Figure 7 a shows the plot of the equibiaxial stress-strain relationship describing the equibiaxial state of the chinchilla TM. The tissue has a linear mechanical behavior within a small range of strain and the mechanical behavior becomes nonlinear after strain becomes larger. The TM tissue becomes stiffer when it is under higher strain. The stiffening effect is evident when the mechanical behavior of the TM is plotted in equibiaxial tangent modulus, which is defined as the derivative of equibiaxial stress with respect to equibiaxial strain from Eqn (6), given as (Thurn, et al., 2004)

$$
\frac{d T_{B}}{d \varepsilon_{B}}=\sum_{i=1}^{N} \frac{2 \mu_{i}}{\alpha_{i}}\left[\left(\alpha_{i}-1\right)\left(1+\varepsilon_{B}\right)^{\alpha_{i}-2}+\left(2 \alpha_{i}+1\right)\left(1+\varepsilon_{B}\right)^{-2 \alpha_{i}-2}\right], i=1,2, \ldots N
$$

The equibiaxial tangent modulus under biaxial stress state for the TM is shown in Figure 7(b). The stiffening effect is observed and the mechanical behavior is linear when the strain is within $0 \%-5 \%$. At strains smaller than $5 \%$, the equibiaxial tangent modulus, which is equal to the equibiaxial modulus, is $52.8 \pm 6.2 \mathrm{MPa}$. 
Since uniaxial tensile test is the most commonly used test for TM measurement, for the purpose to compare our results to others' work, it is convenient to estimate the uniaxial behavior of TM with the parameters in Ogden model. The uniaxial form of $\mathrm{N}$-order Ogden model is given as (Aernouts, et al., 2010; Wang et al., 2002)

$$
T_{U}=\sum_{i=1}^{N} \frac{2 \mu_{i}}{\alpha_{i}}\left(\lambda_{U}^{\alpha_{i}-1}-\lambda_{U}^{-0.5 \alpha_{i}-1}\right), i=1,2, \ldots N
$$

where $T_{U}$ is the uniaxial stress; $\lambda_{U}$ is the uniaxial stretch ratio, and $\lambda_{U}=1+\varepsilon_{U}$, with $\varepsilon_{U}$ being the axial strain. Under uniaxial stretch, assuming incompressibility of the TM, the principal stretch ratios $\lambda_{i}(i=1,2,3)$ are given as $\lambda_{1}=\lambda_{U}, \lambda_{2}=\lambda_{3}=\lambda_{U}{ }^{-0.5}$.

The Young's modulus is defined as the slope in the linear region of the uniaxial stress-strain curve under small deformations. The slope of the stress-strain curve at any point is the tangent modulus. The Young's modulus and the tangent modulus are identical at the initial, linear portion of a stress-strain curve. In the case of a hyperelastic material, the tangent modulus, $E_{\mathrm{t}}$, can be obtained by taking derivative of stress with respect to strain from Eqn

$E_{t}=\frac{d T_{U}}{d \varepsilon_{U}}=\sum_{i=1}^{N} \frac{2 \mu_{i}}{\alpha_{i}}\left[\left(\alpha_{i}-1\right)\left(1+\varepsilon_{U}\right)^{\alpha_{i}-2}+\left(0.5 \alpha_{i}+1\right)\left(1+\varepsilon_{U}\right)^{-0.5 \alpha_{i}-2}\right], i=1,2, \ldots, N(9)$

With known material parameters, Eqn (8) is plotted in Figure 7(c). The uniaxial stress-strain curve of the chinchilla TM, which represents the mechanical behavior under a tensile test, also exhibits a strong nonlinearity. But the trend is different from that under equibiaxial state: the stiffening effect starts at a much higher strain level ( 100\%), which will be higher than the strain $(\sim 31 \%)$ used in the current experiment. In Figure 7(d), the tangent modulus in general decreases with the increase of strain up to $31 \%$ of axial strain. At $31 \%$ strain, which is the maximum strain of the TM determined by FEM model when $1.0 \mathrm{kPa}$ air pressure was applied on the TM, the maximum stress reaches 1.1 MPa. Eqn (9) is plotted in Figure 7(d), showing the tangent modulus as a function of strain for 10 chinchilla TMs, and the average. At a strain close to zero, the average tangent modulus is $25 \mathrm{MPa}$, and it decreases to $11 \mathrm{MPa}$ at $31 \%$. The average tangent modulus, at strains less than $25 \%$, is chosen as the representative Young's modulus. Therefore, the average value of approximately $19 \mathrm{MPa}$ is quoted as the Young's modulus. For comparison equibiaxial tangent modulus and Young's modulus values at different strains are given in Table 2. Under equibiaxial stress state the modulus is consistently higher than the corresponding modulus under uniaxial stress state.

\section{DISCUSSIONS}

The mechanical properties of TM are difficult to measure due to its small size. The traditional measurement using strips cut from the intact TM induces not only damage to the 
TM structures, but also leads to difficulty in controlling the exact dimensions and extension of a sample. For example, in tensile test, the gauge length to width ratio was usually chosen as about 3 (Cheng, et al., 2007), which is less than the standard value used in the material tensile testing (typically 5) (ASTM E8/E8m-13a standard, 2015). Also, it is a challenge to clamp the soft tissue without inducing boundary effects. An in-situ indentation technique was proposed on the TM, to avoid cutting of the TM (Aernouts et al., 2010, 2012a, 2012b, 2012c). However, that approach is a contact method and does not yield the TM mechanical responses under pressure directly. The present approach is a non-contact method, which does not induce any local damage to the TM structures under test; and air pressure is directly applied as loading. Instead of matching directly calculated deformation field with its experimental counterpart at a given pressure. In this work, volume displacement was used to determine the nonlinear mechanical properties of a TM under a series of pressure states from negative pressures to positive pressures. Because the simulations were based on the assumption that chinchilla TM consists of a homogeneous, isotropic material, and its thickness is uniform over the entire pars tensa, the Young's modulus obtained in this study represents an overall effective Young's modulus. In the actual situation, due to the nonuniform distribution of the collagen fibers over TM, the variation of thickness of TM at different locations (Kuypers, et al., 2006; Kuypers, et al., 2005; van der Jeught, et al., 2013) was not considered.

In addition, the mechanical properties can also change with locations. Therefore, even though the model was developed from a 3D surface reconstruction of the specimen, there are still pronounced discrepancies between simulations and experiments at different locations. Shape difference of chinchilla TM between FEM simulations and experiments is given in Figure 8(a) and 8(b), respectively, at two extreme loading cases under $1.0 \mathrm{kPa}$ and $-1.0 \mathrm{kPa}$. Figures $8(\mathrm{c})$ and $8(\mathrm{~d})$ show the error map, which is determined by subtracting the experimental $\mathrm{z}$-height data from the corresponding FEM simulated height data at $1.0 \mathrm{kPa}$ and $-1.0 \mathrm{kPa}$, respectively.

Although at some small local region, a relatively large error as high as $0.15 \mathrm{~mm}$ occurs, most area has error less than $0.017 \mathrm{~mm}$. Overall a reasonably good agreement has been reached. It is seen that discrepancy is generally small in areas with small deformations and high in areas with large deformations. That observation is similar to what was reported in a rat model (Ghadarghadar, et al. 2013). Despite of the discrepancy in local displacements, a good agreement is achieved between computational volume displacements and the corresponding experimental values. Figures 8(a) and 8(b) show comparison of shape profile in Z-height between FEM simulation and experimental data under pressures $1.0 \mathrm{kPa}$ and $-1.0 \mathrm{kPa}$, respectively, along with the profile along the posterior-anterior section shown in black dash line, through umbo, and along superior-inferior shown as red dash line, which is $2.0 \mathrm{~mm}$ from umbo. The small positive and negative errors induced by thickness and properties nonuniformity are reduced through the summation process for the calculation of volume displacement. Therefore in order to reduce the complexity to determine mechanical properties using a hybrid approach, the use of volume displacement for optimization has certain benefit. 
In the simulations, the thickness of TM was set as $10 \mu \mathrm{m}$. The value is the average from the thickness measured at different locations of chinchilla TM based on histology results (Vrettakos, et al., 1988). It is noted that Young's modulus value of TM from FEM analysis is very sensitive to the TM thickness, especially when the thickness is small. The Young's modulus value increases as the assumed thickness of TM decreases. If the thickness of TM was set as $5 \mu \mathrm{m}$, the Young's modulus would be on the $54 \mathrm{MPa}$, which is three times of the current value. This could be responsible for the large scatter in literature data for TM mechanical properties. In literature, TM thickness was usually assumed to be uniform. Nevertheless, the range of chinchilla TM thickness is often measured from $8 \mu \mathrm{m}$ to $12 \mu \mathrm{m}$. Within this range, the variation of Young's modulus is only about 15\%, which is an acceptable range of fluctuation.

Fringe projection was utilized in this study due to its ability for full-field surface topography measurement, high accuracy (about $0.2 \%$ out-of-plane error) (Liang, 2010) and acceptable resolution (about $15 \mu \mathrm{m}$ ) for the relatively large deformation induced by pressure. As compared with other Moiré techniques, such as shadow moiré, projection moiré and reflection moiré, it does not require precise alignment of optical setup. It also does not require an accurate control system for phase-shifting, which is a benefit from a virtual phase-shifting applied on a single experimental image through the computerized processing. The aforementioned benefits show that micro-fringe projection technique also has potential for measurement of dynamic properties with the use of a high speed camera.

Young's modulus data in literature on chinchilla TM is sparse, we therefore compare Young's modulus, obtained in this study with measurement obtained for other animals. For rodent TM, using an indentation technique, Young's modulus of rat was measured as around $21 \mathrm{MPa}$, which is close to the average Young's modulus obtained in this study. Young's moduli of TM of other animals around the size of chinchilla were also reported in a few of papers. Young's modulus of rabbit TM was reported as $30 \mathrm{MPa}$ (Aernouts, et al., 2010); and for cat the Young's modulus was estimated as 100 400 MPa (Fay, et al., 2005). The diameter of chinchilla TM is similar to human TM. For human, Young's moduli were reported under different situations. The $20 \mathrm{MPa}$ was reported under an in-vivo condition (von Békésy, 1960), similar values were obtained on TM samples along radial and circumferential directions (Luo, et al., 2009a, 2009b) under high strain rates. The Young's modulus of human TM measured by uniaxial tension of strips cut from TM was measured to be at $10 \mathrm{MPa}$ and $23 \mathrm{MPa}$, respectively (Decraemer, et al., 1980; Kirikae, 1960). It is noted that, cutting TM breaks the collagen fibers, which subsequently shrink. This could induce variations from one specimen to another, causing a large variation of measurement results. Direct measurement of the mechanical properties from the entire pars tensa TM sample can maintain the integrity of TM and yields more reliable data. To the best of our knowledge there was only one study on full-field intact rat TM reported (Ghadarghadar, et al., 2013). Young's storage and loss modulus values of intact TM have been measured at frequency 0.1 $\mathrm{Hz}$, they are $40 \mathrm{MPa}$ and $19 \mathrm{MPa}$, respectively at frequency $0.1 \mathrm{~Hz}$ (Ghadarghadar, et al., 2013). The results from this study show that the Young's modulus of chinchilla TM is in the range of $11 \sim 30 \mathrm{MPa}$ at strains below $25 \%$, slightly higher than the reported values for human TM. While data has been reported for elastic coefficients determined from numerous TMs, including human TM and guinea pig TM (Békéy, 1949; Gan, et al., 2010, 2013; Guan, 
et al., 2013; Zhang, et al., 2013a, 2013b), the stress-strain curve of chinchilla TM was not reported in literature, especially when it is measured from the intact TM. This study fills the gap by providing the nonlinear stress-strain relationship, including Young's modulus of chinchilla TM, measured at $19.0 \pm 4.5 \mathrm{MPa}$, and tangent modulus at strains up to $31 \%$.

\section{CONCLUSION}

The nonlinear mechanical response of chinchilla TM was determined using micro-fringe projection technique. Quasi-static air pressure was applied to a chinchilla TM at the lateral side through the ear canal inside the bulla. A micro-fringe projection method was used to measure the surface topography of the TM. The volume displacement of the TM was used as input to a finite element model for simulation. The Ogden hyperelastic model was used to describe the constitutive behavior of the TM in FEM for simulations. An inverse method was used to allow the pressure-volume displacement relationship simulated by FEM to match with the experimental results. The model parameters were then used to describe the mechanical behavior of chinchilla TM, and stress-strain curve under both equibiaxial and uniaxial stress states. Under equibiaxial stress state, the equibiaxial tangent modulus increases with the increase of strain, while under uniaxial stress state, the tangent modulus decreases with the increase of strain. The Young's modulus of the chinchilla TMs was determined to be approximately $19 \mathrm{MPa}$, up to a strain level of $25 \%$. As strain increases from 0 to $31 \%$, the tangent modulus decreases from $25 \mathrm{MPa}$ to $11 \mathrm{MPa}$. The maximum stress experienced by the TM used in these experiments reaches 1.1 MPa.

\section{Acknowledgments}

We acknowledge the support of NIH R01DC011585, DOD W81XWH-14-1-0228, DOD W81XWH-13MOMJPC5-IPPEHA, and NSF CMMI-1031829, CMMI-1132174, ECCS-1307997 and NSF IIP-1522559. Lu acknowledges the Louis A. Beecherl Jr., Chair for additional support.

\section{List of the Abbreviations}

A

CAD

FEM finite element method

I

IACUC Institutional Animal Care and Use Committee

IM intramuscular

LDV laser Doppler vibrometery

$\mathbf{P} \quad$ posterior

PVC polyvinyl chloride

S superior 
TM tympanic membrane

U umbo

XYZ $\quad \mathrm{X}-, \mathrm{y}-$ and z-axes

\section{References}

Aernouts J, Soons JAM, Dirckx JJJ. Quantification of tympanic membrane elasticity parameters from in situ point indentation measurements: Validation and preliminary study. Hearing Res. 2010; 263:177-182.

Aernouts J, Dirckx JJJ. Static versus dynamic gerbil tympanic membrane elasticity: derivation of the complex modulus. Biomech Model Mechan. 2012a; 11:829-840.

Aernouts J, Dirckx JJJ. Viscoelastic properties of gerbil tympanic membrane at very low frequencies. J Biomech. 2012b; 45:919-924. [PubMed: 22326125]

Aernouts J, Aerts JRM, Dirckx JJJ. Mechanical properties of human tympanic membrane in the quasistatic regime from in situ point indentation measurements. Hearing Res. 2012c; 290:45-54.

Aerts JRM, Dirckx JJJ. Nonlinearity in eardrum vibration as a function of frequency and sound pressure. Hearing Res. 2010; 263:26-32.

ASTM E8/E8M-13aStandard Test Methods for Tension Testing of Metallic MaterialsASTM International; West Conshohocken, PA: 2015

Békéy GV. The structure of the middle ear and the hearing of one's own voice by bone conduction. J Acoust Soc Am. 1949; 21:217-232.

Buytaert JAN, Dirckx JJJ. Tomographic imaging of macroscopic biomedical objects in high resolution and three dimensions using orthogonal-plane fluorescence optical sectioning. Appl Optics. 2009; 48:941-948.

Cheng T, Dai CK, Gan RZ. Viscoelastic properties of human tympanic membrane. Ann Biomed Eng. 2007; 35:305-314. [PubMed: 17160465]

Daphalapurkar NR, Dai CK, Gan RZ, Lu HB. Characterization of the linearly viscoelastlic behavior of human tympanic membrane by nanoindentation. J Mech Behav Biomed. 2009; 2:82-92.

Decraemer WF, Maes MA, Vanhuyse VJ. An elastic stress-strain relation for soft biological tissues based on a structural model. J Biomech. 1980; 13:463-468. [PubMed: 7400174]

Dirckx JJJ, Decraemer WF. Human tympanic membrane deformation under static pressure. Hearing Res. 1991; 51:93-106.

Dirckx JJJ, Decraemer WF. Coating techniques in optical interferometric metrology. Appl Optics. 1997; 36:2776-2782.

Fay J, Puria S, Decraemer WF, Steele C. Three approaches for estimating the elastic modulus of the tympanic membrane. J Biomech. 2005; 38:1807-1815. [PubMed: 16023467]

FungYC. Biomechanics: Mechanical Properties of Living Tissues2. Springer; New York: 1993

Funnell WRJ, Decraemer WF. On the incorperation of Moire shape measurements in finite-element models of the cat eardrum. J Accoust Soc Am. 1995; 100:925-932.

Gaihede M, Liao D, Gregersen H. In vivo arealmodulus of elasticity estimation of the human tympanic membrane system: modelling of middle ear mechanical function in normal young and aged ears. Phys Med Biol. 2007; 52:803-814. [PubMed: 17228122]

Gan R, Dai C, Wang X, Nakmali D, Wood MW. A totally implantable hearing system - Design and function characterization in 3D computational model and temporal bones. Hearing Res. 2010; 263:138-144.

Gea SLR, Decraemer WF, Funnell WRJ, Dirckx JJ, Maier H. Tympanic membrane boundary deformations derived from static displacements observed with computerize tomography in human and gerbil. J Assoc Res Otolaryngol. 2010; 11:1-17. [PubMed: 19834763]

Ghadarghadar N, Agrawal SK, Samani A, Ladak HM. Estimation of the quasi-static Young's modulus of the eardrum using a pressurization technique. Comput Meth Prog Bio. 2013; 110:231-239. 
GhigliaDC, , PrittMD. Two-dimensional Phase Unwrapping, Theory, Algorithms, and SoftwareJohn Wiley and Sons; New York: 1998

De Greef D, Soons J, Dirckx JJJ. Digital stroboscopic holography setup for deformation measurement at both quasi-static and acoustic frequencies. Int J Optomech. 2014a; 8:275-291.

De Greef D, Aernouts J, Aerts J, Cheng JT, Horwitz R, Rosowski JJ, Joris JJ, Dirckx JJJ. Viscoelastic properties of the human tympanic membrane studied with stroboscopic holography and finite element modeling. Hearing Res. 2014b; 312:69-80.

Guan X, Gan R. Mechanisms of tympanic membrane and incus mobility loss in acute otitis media model of guinea pig. J Assoc Res Otolaryngol. 2013; 14:295-307. [PubMed: 23483330]

Hesabgar SM, Marshall H, Agrawal SK, Samani A, Ladak HM. Measuring the quasi-static Young's modulus of the eardrum using an indentation technique. Hearing Res. 2010; 263:168-176.

Huang G, Daphalapurkar NP, Gan RZ, Lu HB. A method for measuring linearly viscoelastic properties of human tympanic membrane using nanoindentation. J Biomech Eng-Trans ASME. 2008; 130:014501.

KirikaeI. The Structure and Function of Middle EarUniversity of Tokyo Press; Tokyo: 1960

Kuypers LC, Decraemer WF, Dirckx JJJ, Timmermans JP. Thickness distribution of fresh eardrums of cat obtained with confocal microscopy. J Assoc Res Otolaryngol. 2005; 6:223-233. [PubMed: 15983727]

Kuypers LC, Decraemer WF, Dirckx JJJ. Thickness distribution of fresh and preserved human eardrums measured with confocal microscopy. Otol Neurotol. 2006; 27:256-264. [PubMed: 16436998]

Ladak HM, Decraemer WF, Dirckx JJJ, Funnell WRJ. Response of the cat eardrum to static pressures: Mobile versus immobile malleus. J Acoust Soc Am. 2004; 116:3008-3021. [PubMed: 15603146]

Ladak HM, Funnell WRJ, Decraemer WF, Dirckx JJJ. A geometrically nonlinear finite-element model of the cat eardrum. J Acoust Soc Am. 2006; 119:2859-2868. [PubMed: 16708944]

Lee CY, Rosowski JJ. Effects of middle-ear static pressure on pars tensa and pars flaccida of gerbil ears. Hearing Res. 2001; 153:146-163.

LiangJ. Master ThesisOklahoma State University; Stillwater, OK, USA: 2010Determination of the mechanical properties of guinea pig tympanic membrane using combined fringe projection and simulations.

Luo H, Dai C, Gan R, Lu H. Measurement of Young's modulus of human tympanic membrane at high strain rates. J Biomech Eng-Trans ASME. 2009a; 131:064501.

Luo H, Lu H, Dai C, Gan R. A comparison of Young's modulus for normal and diseased human eardrums at high strain rates. Int J Exp Comput Biomech. 2009b; 1:1-22.

Luo H, Jiang S, Nakmali DU, Gan RZ, Lu H. Mechanical properties of a human eardrum at high strain rates after exposure to blast waves. J Dynamic Behavior Mater. 2016; 2:59-73.

O'Connor KN, Tam M, Blevins NH, Puria S. Tympanic membrane collagen fibers: A key to highfrequency sound conduction. Laryngoscope. 2008; 118:483-490. [PubMed: 18091335]

Ogden RW. Large deformation isotropic elasticity - On the correlation of theory and experiment for incompressible rubberlike solids. Proc Roy Soc Lon- A, Mathemat Phys Sci. 1972; 326:565-584.

Ortiz MH, Patterson EA. On the industrial applications of Moire and fringe projection techniques. Strain. 2003; 39:95-100.

OrtizMH. Novel Development of Moire Techniques for Industrial ApplicationUniversity of Sheffield; 2004

Ortiz MH, Patterson EA. Location and shape measurement using a portable fringe projection system. Exp Mech. 2005; 45:197-204.

Ritenour AE, Wickley A, Ritenour JS, Kriete BR, Blackbourne LH, Holcomb JB, Wade CE. Tympanic membrane perforation and hearing loss from blast overpressure in operation enduring freedom and operation Iraqi freedom wounded. J Trauma. 2008; 64:174-178. [PubMed: 18188118]

Rosowski JJ, Lee CY. The effect of immobilizing the gerbil's pars flaccida on the middle-ear's response to static pressure. Hearing Res. 2002; 174:183-195. 
RosowskiJJ, , NakajimaHH, , ChengJT. Current topics in the study of sound conduction to the inner ear, Chapter 26 in Perspectives on Auditory Research. In: PopperAN, , FayRR, editorsHandbook of Auditory ResearchVol. 50. Springer; 2014493511

Salamati E, Agrawal SK, Samani A, Ladak HM. Estimation of the othotropic elastic properties of the rat eardrum. J Med Biol Eng. 2012; 32:225-234.

Soons JAM, Aernouts J, Dirckx JJJ. Elasticity modulus of rabbit middle ear ossicles determined by a novel micro-indentation technique. Hearing Res. 2010; 263:33-37.

Thornton JL, Chevallier KM, Koka K, Gabbard SA, Tollin D. Conductive hearing loss induced by experimental middle-ear effusion in a chinchilla model reveals impaired tympanic membranecouple ossicular chain movement. J Assoc Res Otolaryngo. 2013; 14:451-465.

Thurn J, Hughey MP. Evaluation of film biaxial modulus and coefficient of thermal expansion from thermoelastic film stress measurements. J Appl Phys. 2004; 95:7892.

Van der Jeught S, Dirckx JJJ, Aerts JRM, Bradu A, Podoleanu AG, Buytaert JAN. Full-field thickness distribution of human tympanic membrane obtained with optical coherence tomography. J Assoc Res Otolaryngol. 2013; 14:483-494. [PubMed: 23673509]

Volandri G, Di Puccio F, Forte P, Carmignani C. Biomechanics of the tympanic membrane. J Biomech. 2011; 44:1219-1236. [PubMed: 21376326]

von BékésyG. Experiments in HearingMcGraw-Hill Book Company; New York: 1960

von Unge M, Decraemer WF, Baggersjoback D, Dirckx JJ. Displacement of the gerbil tympanic membrane under static pressure variations measured with a real-time differential Moire interferometer. Hearing Res. 1993; 70:229-242.

von Unge M, Dircks JJ. Functional effects of repeated pressure loads upon the tympanic membrane: mechanical stiffness measurements after simulated habitual sniffing. Eur Arch Oto-Rhino-L. 2009; 266:1219-1224.

Vrettakos PA, Dear SP, Saunders JC. Middle ear structure in the chinchilla: A quantitative study. Am J Otolaryngology. 1988; 9:58-67.

Wang B, Lu H, Kim G. A damage model for the fatigue life of elastomeric materials. Mech Mater. $2002 ; 34: 475-483$.

Wang X, Cheng T, Gan R. Finite-element analysis of middle-ear pressure effects on static and dynamic behavior of human ear. J Acoust Soc Am. 2007; 122:906-917. [PubMed: 17672640]

Zhang X, Gan R. Dynamic properties of human tympanic membrane - Experimental measurement and modelling analysis. Int J Exp Comput Biomech. 2010; 1:252-270.

Zhang X, Gan R. Finite element modeling of energy absorbance in normal and disordered human ears. Hearing Res. 2013a; 301:146-155.

Zhang X, Gan R. Dynamic properties of human tympanic membrane based on frequency-temperature superposition. Ann Biomed Eng. 2013b; 41:205-214. [PubMed: 22820983] 


\section{Highlights}

- Mechanical behavior of chinchilla TM was characterized under pressure loading.

- $\quad$ TM topography in reference/deformed state was determined by micro-fringe projection.

- Volume displacement was determined as a function of positive or negative pressure.

- $\quad$ Finite element implementing hyperelastic model was used to simulate TM response.

- Young's moduli of chinchilla TM was determined as 19 MPa up to a strain level of $25 \%$. 
(a)

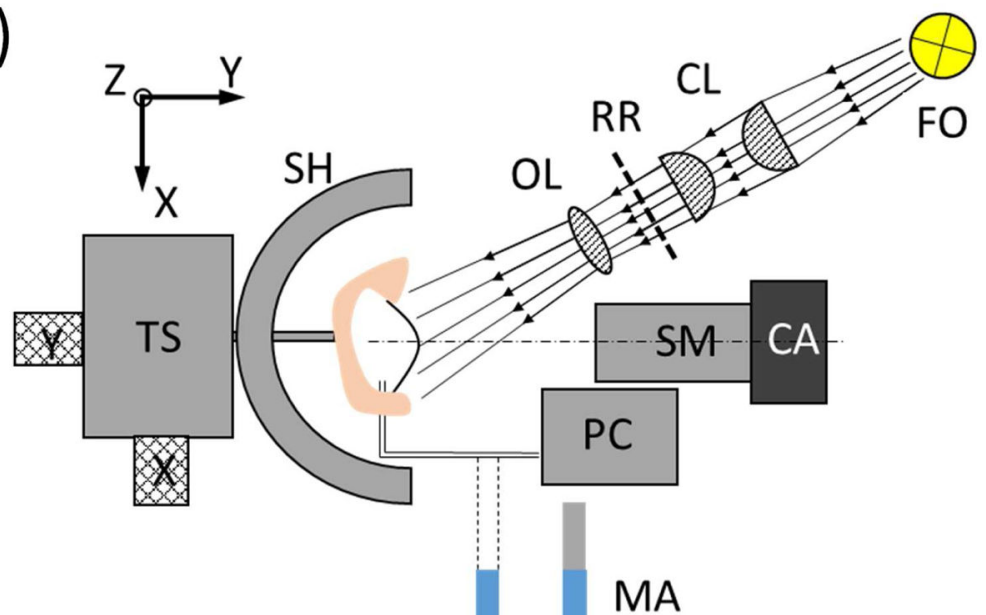

(b)

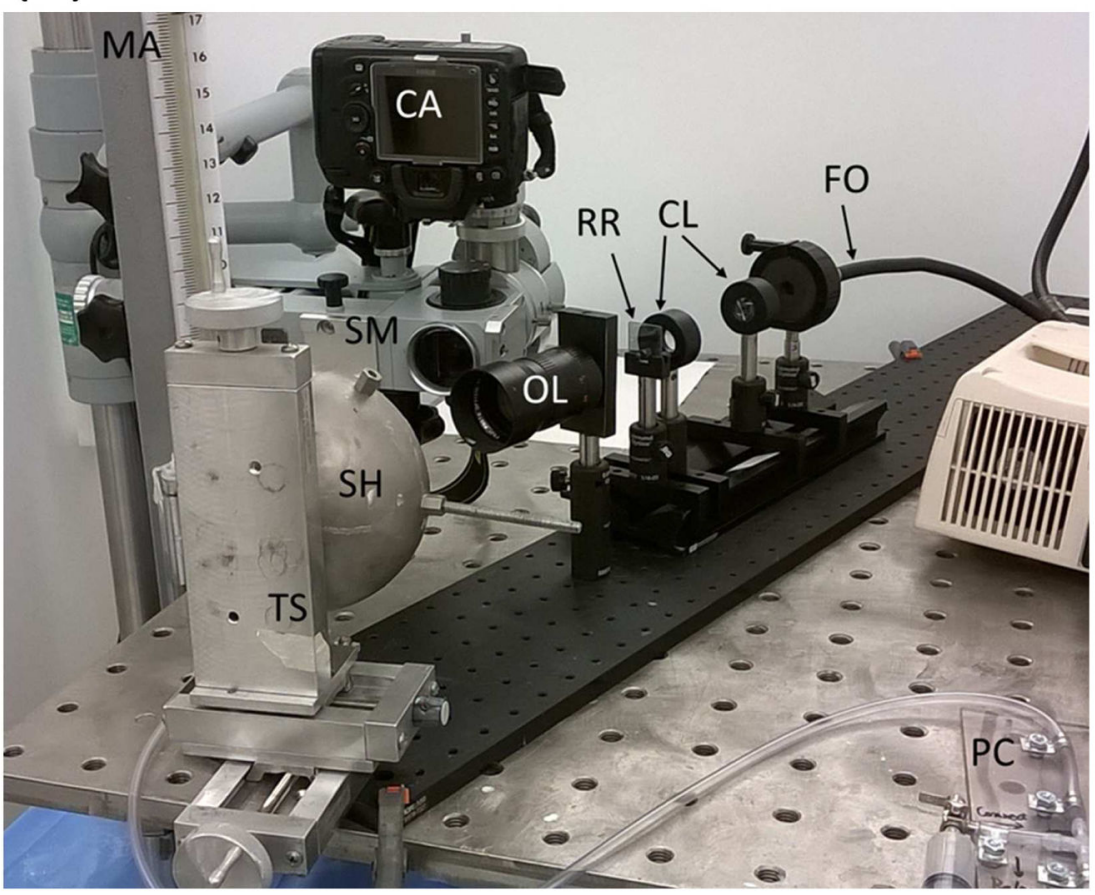

Figure 1.

The experimental setup of the micro-fringe projection system and the pressure loading and monitoring system. (a) Schematic diagram; (b) Actual setup. The components include: manometer (MA), camera (CA), surgical microscope (SM), sample holder (SH), XYZtranslation stage (TS), objective lens (OL), Ronchi rulings (RR), condenser lenses (CL), fiber optics illuminator (FO) and pressure control (PC). 


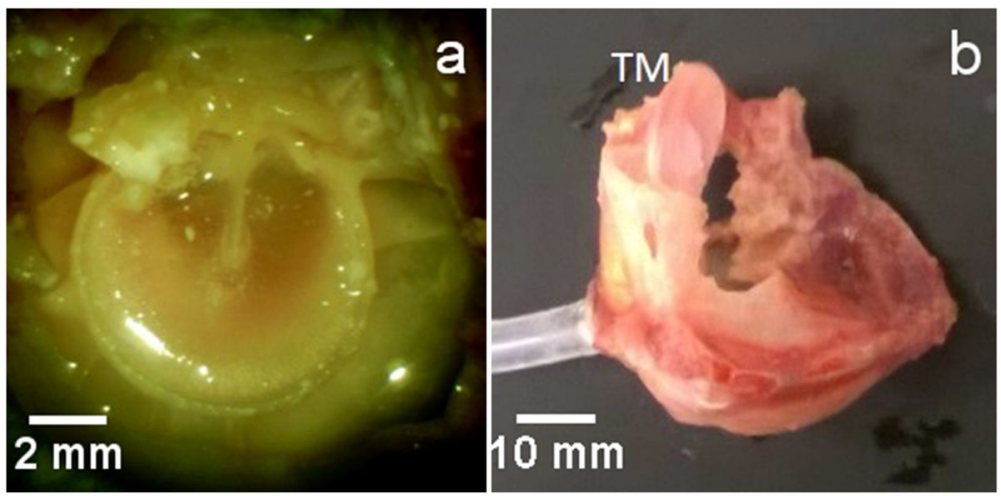

Figure 2.

A chinchilla bulla sample for measurements: (a) The medial side of TM with intact malleusincus complex immobilized; (b) A typical bulla sample with TM exposed, and a sealed PVC tube inserted into ear canal for applying pressure. 

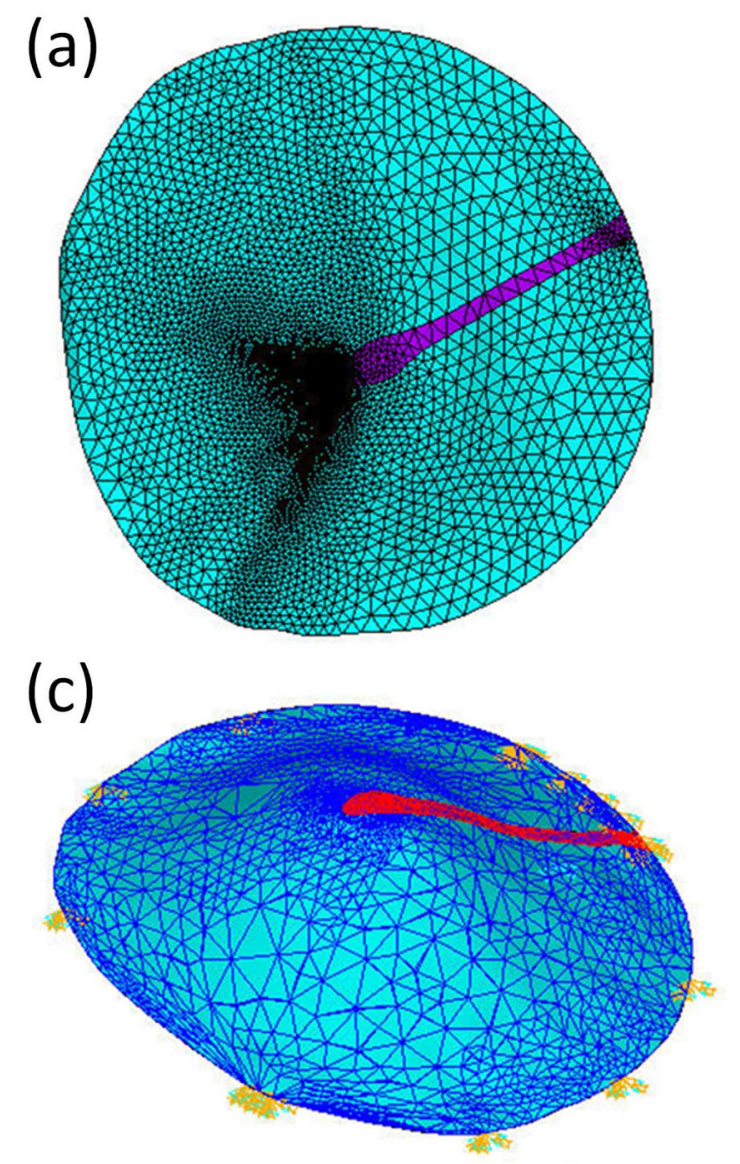

(b)

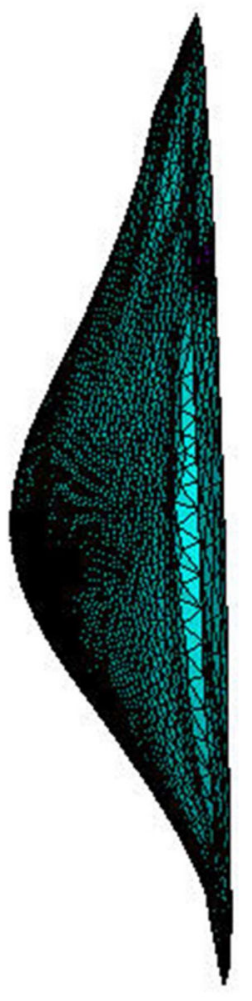

Figure 3 .

Finite element model of chinchilla TM in different views. The model consists of 10,000 shell elements (shell-181). The annular ring of the TM is fixed for all degrees of freedom. The area marked in red color is the manubrium whose material properties are set as those (Young's modulus, etc.) for bones. (a). Medial-view of meshed TM; (b). Posterior view of meshed TM; (c). Boundary conditions for TM, as shown with yellow arrows. 

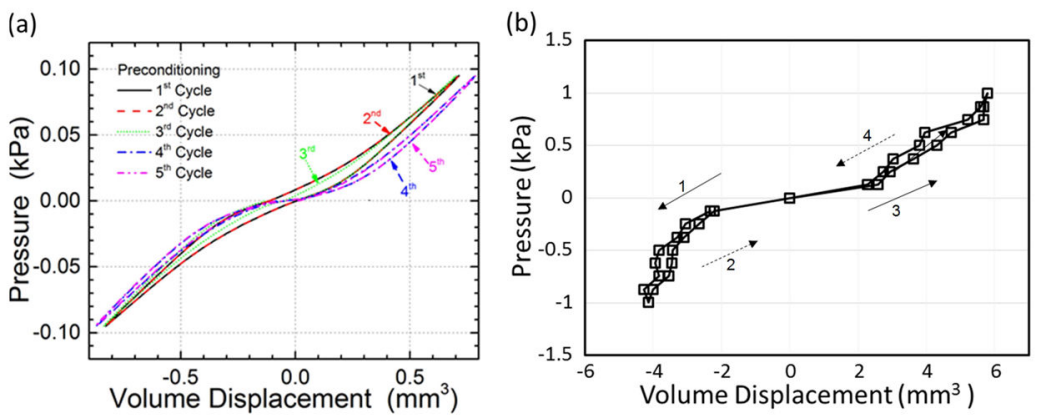

Figure 4.

Pressure-volume displacement curves. (a) Typical 5 cycles of preconditioning for a chinchilla TM. (b) Typical one cycle of loading and unloading. The sequence of loading is as follows: negative pressure was first applied on the TM (creating a vacuum from ear canal side) up to $-0.1 \mathrm{kPa}$ and then released to zero; subsequently, positive pressure was applied onto TM (pumping air on the ear canal side) up to $0.1 \mathrm{kPa}$ and then released to zero. The loading sequence is $1-2-3-4$ as shown by the arrows. 
(a)

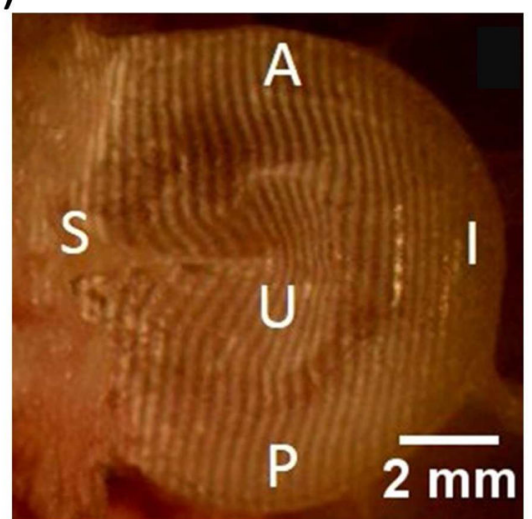

Figure 5. inclination in the reconstructed surface profile. (b)

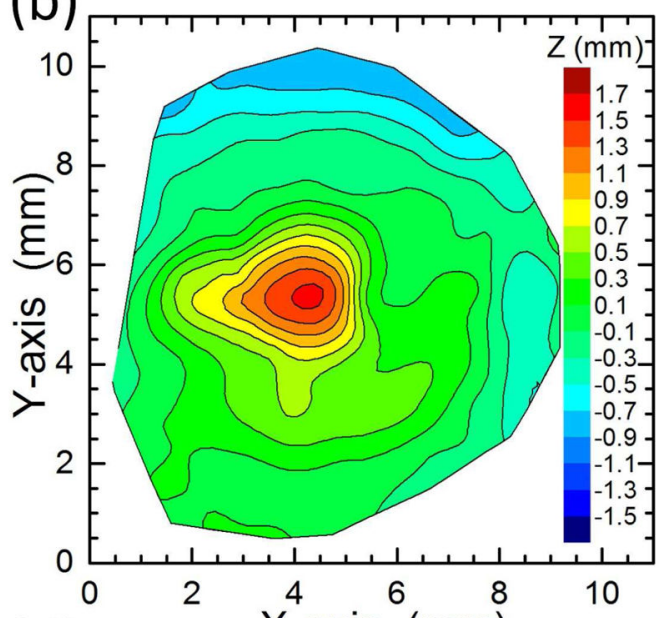

(d)

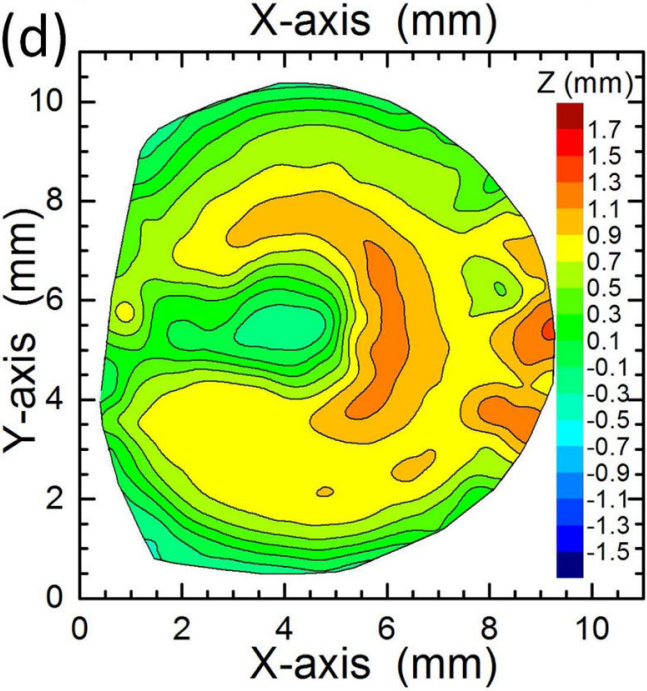

Measurements of projected micro-fringes for chinchilla TM surface topography. (a). Microprojected fringes on a chinchilla TM; (b). Height profile $\mathrm{z}(\mathrm{x}, \mathrm{y})$ under zero pressure; (c). Zdisplacement $\mathrm{U}_{3}$ under $-1.0 \mathrm{kPa}$ pressure (medial side pressure is higher than lateral side pressure); (d). Z-displacement $\mathrm{U}_{3}$ under $1.0 \mathrm{kPa}$ pressure (lateral side pressure is higher than medial side pressure). Note, the reference plane is not necessarily coincident with the annular ring because the annular ring is not on the same plane. Thus there is a slight 
(a)

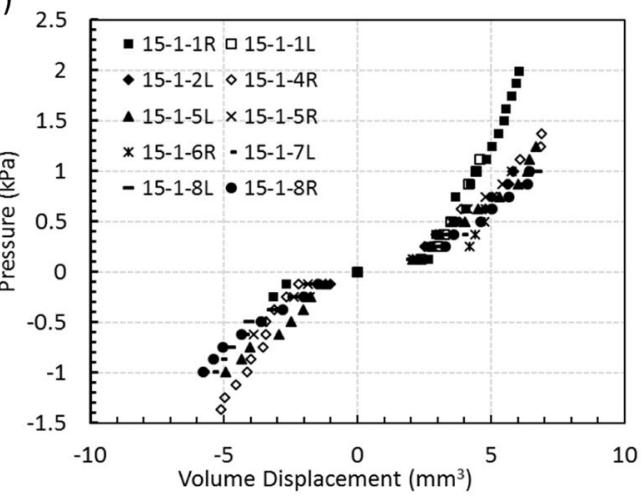

(b)

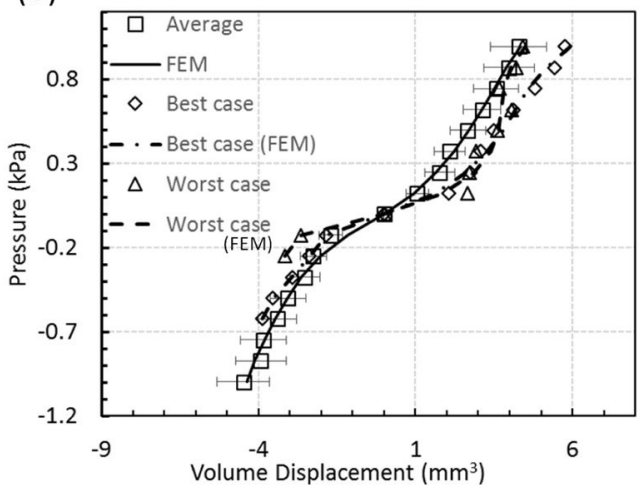

Figure 6.

Pressure-volume displacement relationships for ten chinchilla TMs under different pressures measured with the micro-fringe projection system. (a). Experimental results for10 TMs; (b). A comparison between the finite element simulation results and experimental data. The error bar represents the standard deviation from the experiments. Both best and worst fitting results are given in 6(b). 
(a)

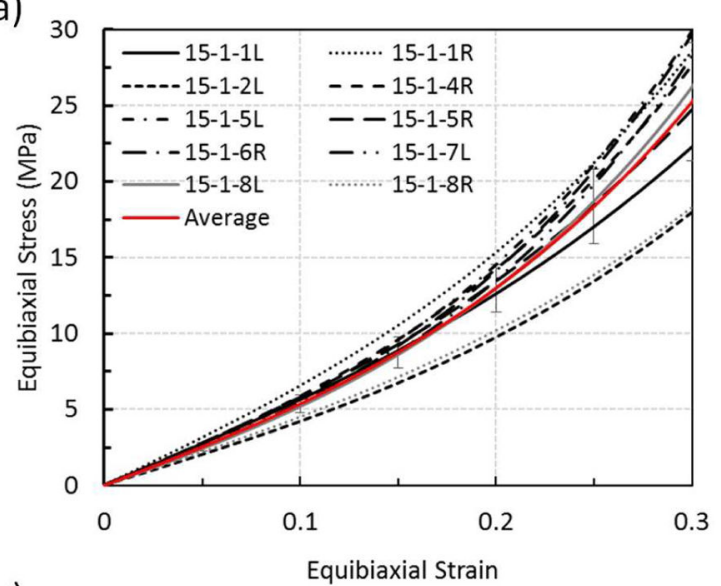

(c)

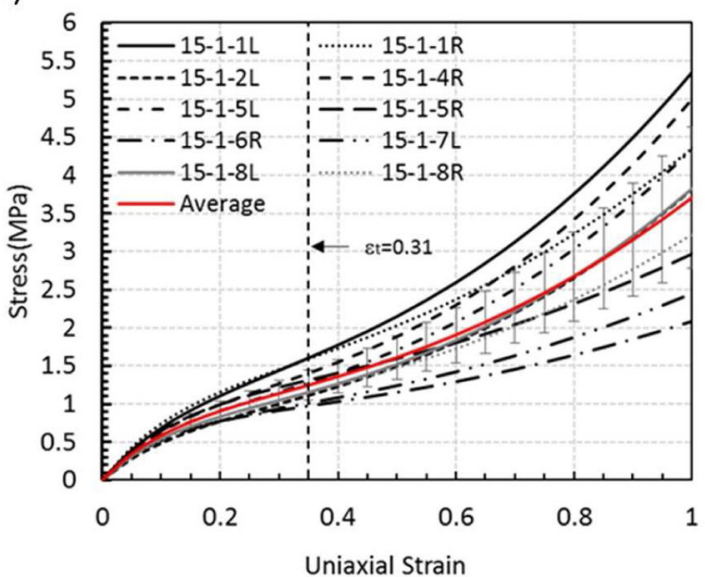

(b)

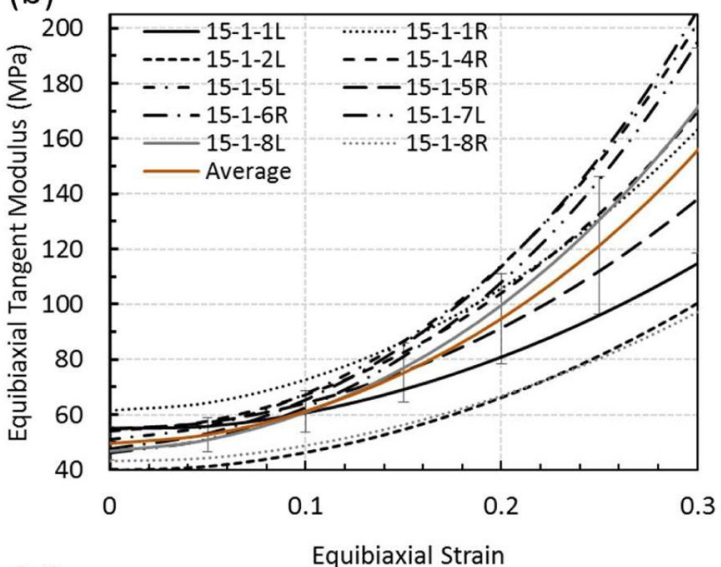

(d)

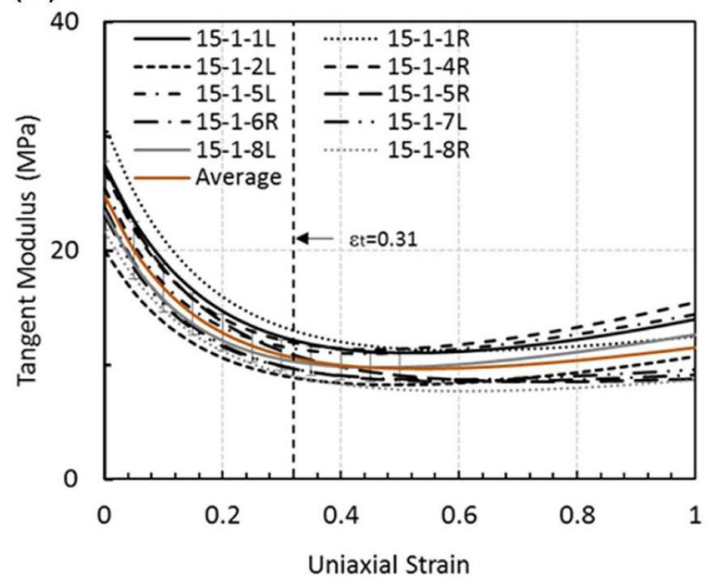

Figure 7.

Mechanical response and mechanical properties of all ten chinchilla TMs derided from the FEM analysis using a second order Ogden model under: (a) Equibiaxial stress-strain curves;

(b) Biaxial modulus; (c) Uniaxial tensile stress-strain curves; (d) Tangent modulus of chinchilla TMs. The error bar represents standard deviation. The dash line in (c) and (d) shows the actual maximum strain in the experiment. 

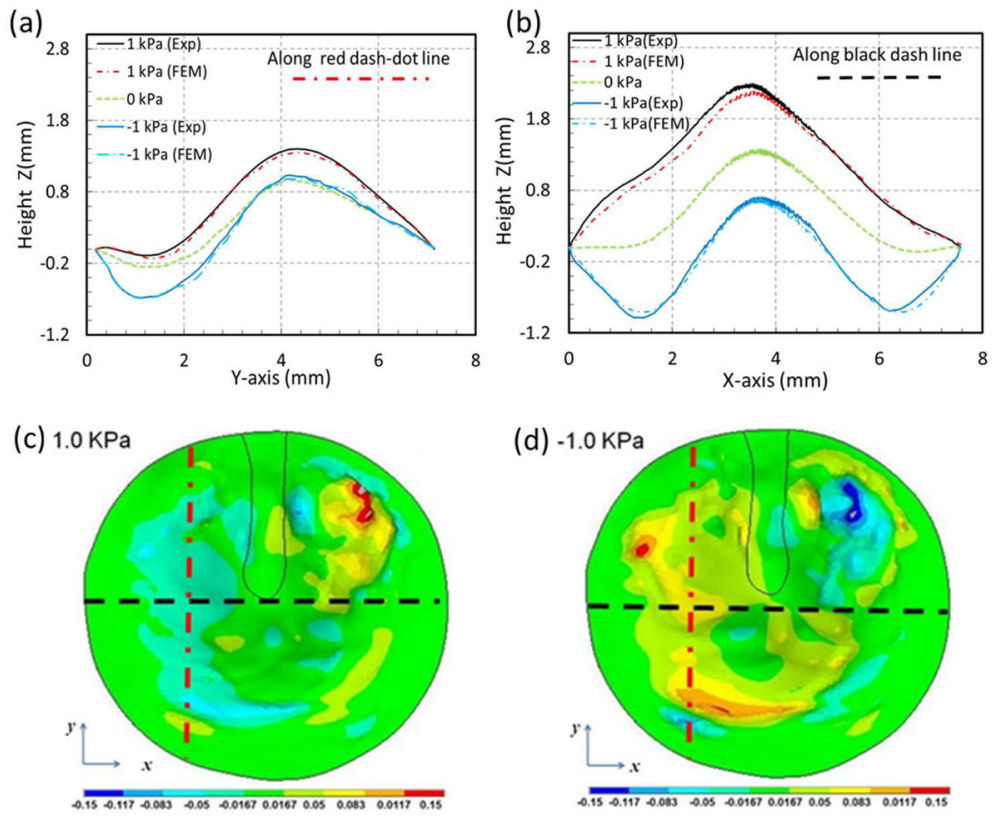

Figure 8.

A comparison of shape profile calculated as z-coordination height between FEM simulation and experimental data at $\pm 1.0 \mathrm{kPa}$. (a). The experimental and FEM simulated $\mathrm{Z}$ height profiles along posterior-anterior section (as shown black dash line, through umbo); (b). Experimental and FEM simulated $\mathrm{Z}$ height profiles along superior-inferior (as shown red dash line, $2.0 \mathrm{~mm}$ off umbo). (c) Error map (FEM simulated map of height subtracted by experimental map of height) at $1.0 \mathrm{kPa}$; (d) Error map at $-1.0 \mathrm{kPa}$; A reasonably good agreement has been reached. 


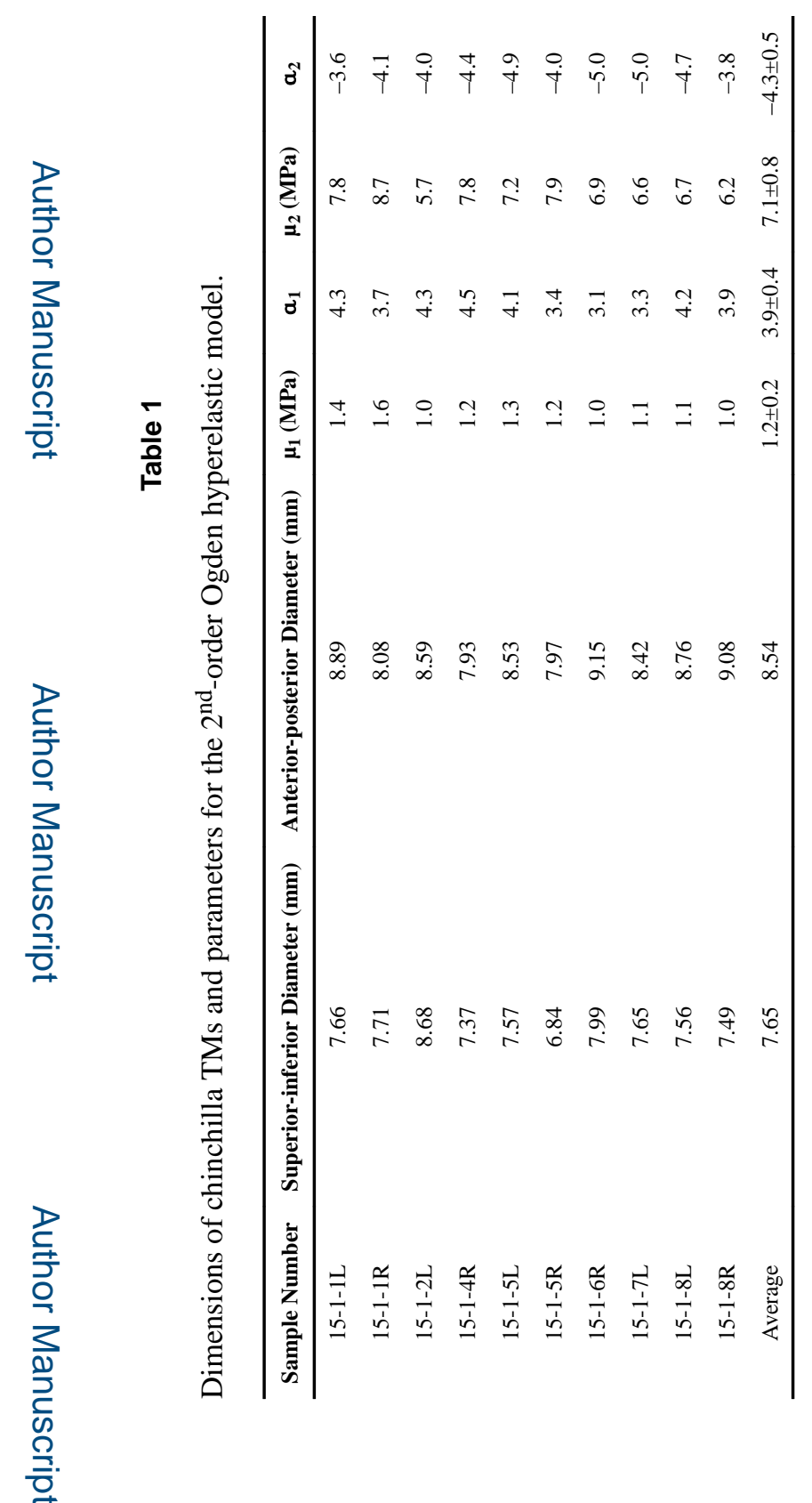

Hear Res. Author manuscript; available in PMC 2018 July 25. 

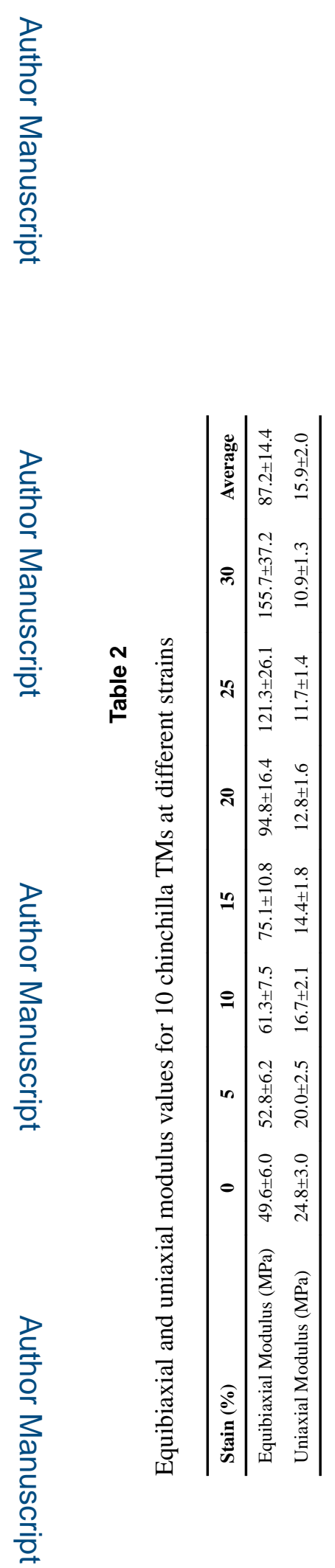

Hear Res. Author manuscript; available in PMC 2018 July 25. 